\title{
Eclipsing binaries and the mass-luminosity relation
}

\author{
O. Yu. Malkov^ \\ Institute of Astronomy of the Russian Acad. Sci. 48 Pyatnitskaya St., Moscow 119017, Russia \\ Observatoire de Besançon, 41bis avenue de l'Observatoire, 25010 Besançon, Cedex, France \\ Isaac Newton Institute of Chile Moscow Branch \\ Received 20 January 2003 / Accepted 25 February 2003

\begin{abstract}
We have compared radii of eclipsing binary components and single stars. We have found a noticeable difference for B0V-G0V components of eclipsing binaries and single stars of the corresponding spectral type. This difference can be confirmed by a re-analysis of results of other published investigations and, in particular, it can explain the disagreement between published scales of bolometric corrections.

According to our results, A- and F-type main sequence eclipsing binaries have larger radii and/or higher temperatures than single stars while B-type eclipsing binaries have smaller radii. Possible explanations for these features are proposed.

We have concluded that the mass-luminosity relation based on empirical data of eclipsing binary components cannot be used to derive the stellar initial mass function. While our current knowledge of the empirical mass-luminosity relation for masses more than $1.5 m_{\odot}$ is based exclusively on eclipsing binaries data, accurate observational data for a few hundred visual binaries of intermediate and high masses should be collected. Then the initial mass function for this mass range should be revised.
\end{abstract}

Key words. stars: binaries eclipsing - stars: luminosity fonction, mass fonction

\section{Introduction}

The relation between the mass of a star and its luminosity on the main sequence (mass-luminosity relation, MLR) is a fundamental law that is used in various fields of astrophysics. It is especially important for the construction of the initial mass function (IMF) from the luminosity function of stars.

Independent stellar mass and luminosity determination is possible only for components of some types of binary systems. Visual binaries with known trigonometric parallax provide masses of components (see Delfosse et al. 2000; Henry et al. 1999; Malkov et al. 1997 for recent low-mass mass-luminosity relations based on visual binaries data). Another main source is detached main-sequence eclipsing binaries (hereafter EB), with the spectrum lines of the two components. Recent massluminosity relations based on eclipsing binaries data can be found, e.g., in Kovaleva (2001), Gorda \& Svechnikov (1998).

To match the two mass-luminosity relations (and also to compare theoretical mass-luminosity relations with empirical data) we made the natural assumption that components of wide and close binaries evolve similarly.

In the present paper we discuss a difference between EB components of detached main sequence systems and main sequence isolated stars (hereafter we use the term "isolated star" for single stars and components of wide binaries).

Observational evidence for a difference in the parameters of EB components from those of isolated stars are given in Sect. 2.

\footnotetext{
* e-mail:malkov@inasan.ru
}

Possible reasons for the difference are discussed in Sect. 3. We discuss the contribution of eclipsing binaries data to the MLR and IMF determination process in Sect. 4, and, finally, in Sect. 5 we draw conclusions.

\section{Comparison of parameters of eclipsing binaries and isolated stars}

In this section we list some examples of observational evidence of a difference between main sequence EB components and isolated stars.

\subsection{Radii}

We have made a comparison of radii of single stars, determined by photometric techniques and collected in the 3rd Edition of the Catalog of Apparent Diameters and Absolute Radii of Stars (CADARS, Pasinetti-Fracassini et al. 2001) with radii of unevolved EB components listed in the Catalogue of astrophysical parameters of binary systems (Malkov 1993).

The following conclusions can be drawn from the comparison (see Fig. 1):

- B-type components of EB show, on average, 20\% smaller radii or/and earlier spectral types (i.e., higher temperatures) than the corresponding isolated stars.

- Contrary, A- and F-type EB components have larger radii than the corresponding isolated stars. 


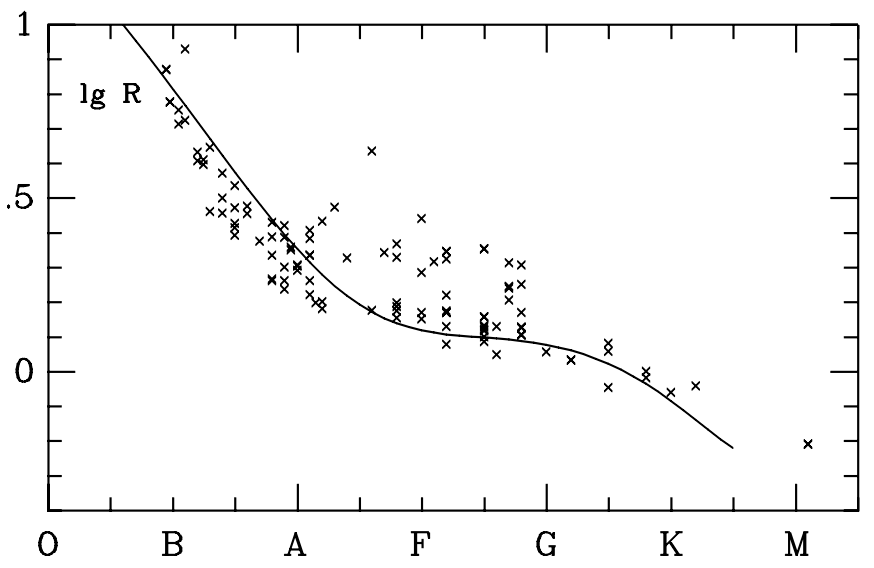

Fig. 1. Radii vs. spectral type for unevolved eclipsing binary components (points) and main sequence single stars (line). Eclipsing binary data (114 components) are taken from the Catalogue of astrophysical parameters of binary systems (Malkov 1993). The typical standard error in their radii is $0.05 R_{\odot}$. Radii of some 3500 main sequence single stars, determined by photometric methods, are taken from the CADARS (Pasinetti-Fracassini et al. 2001) and approximated by a spline. Standard error in radius for CADARS stars is $5-15 \%$.

\subsection{Effective temperatures}

Other confirmations of a difference between the observed parameters of isolated stars and those of EB components can be found in the literature.

Effective temperatures of detached eclipsing binaries computed through Hipparcos trigonometric parallaxes were compared by Ribas et al. (1998) with the photometric determinations. The former are based on the values of the radius $(R)$, the apparent visual magnitude $(V)$ and the bolometric correction of the star:

$T_{\text {eff }}($ Hip $)=T_{\text {eff } \odot} \sqrt{\frac{1}{10 \pi} \frac{R_{\odot}}{R}} \times 10^{-0.1\left(V-A_{V}+B C-M_{\text {bol } \odot}\right)}$,

where $\pi$ is trigonometric parallax, $B C$ is bolometric correction, and $A_{V}$ is interstellar extinction. The photometric temperatures are obtained from standard calibrations using Strömgren or Johnson colour indices. The working sample contained 20 well-studied detached double-lined eclipsing binaries (18 of them were main-sequence systems) belonging to the Hipparcos catalogue and with relative errors in the parallaxes smaller than $20 \%$.

A systematic trend is observed between the two temperature determinations (see Ribas et al.'s Fig. 2) that, as the authors supposed, could be due to inaccuracies in the photometric effective temperatures.

Another possible explanation of this feature, in our opinion, may be the difference of observational parameters between EB components of detached main sequence systems (belonging to the Ribas et al.'s sample) and main sequence isolated stars (standard calibrations used by Ribas et al. are based on data for isolated stars). From this point of view, Ribas et al.'s results (see their Fig. 2b) can lead to the following conclusion:

$T_{\text {eff }}$ (Hip), given by Eq. (1), is systematically smaller than the photometric temperature (obtained from standard calibrations using colour indices) for A0 to at least F8 spectral type
EB components. It means that A and F EB stars have larger radii or/and higher temperatures in comparison with corresponding isolated stars. Larger radii of EB stars lead, according to Eq. (1), to smaller values of their $T_{\text {eff }}($ Hip). Higher temperatures (and consequently bluer color indices) of EB components lead to an increase of the calculated photometric temperature.

No definite conclusion can be drawn for earlier type stars since they are more distant, and so they show a larger scatter.

\subsection{Radiative fluxes}

A similar investigation was made by Popper (1998), who found that the radiative fluxes of components of eclipsing binaries with Hipparcos parallaxes fall well below the curve for single stars with angular diameters.

To compare the fluxes, Popper (1998) used 14 nearby $(d<$ $125 \mathrm{pc}$ ) detached main sequence EB systems with Hipparcos parallax of $10 \%$ or better accuracy. All of these systems have both a spectroscopic and photometric orbital analysis leading to reliable values of the radii, magnitude difference, and color indices of the components. Five of the 14 systems were also used by Ribas et al. (1998) in their effective temperature analysis (see Sect. 2.2).

The radiative fluxes for these stars were calculated according to

$F_{V}=-0.1(V+5+5 \log \pi)-0.5 \log \frac{R}{R_{\odot}}+4.225$,

and plotted as a function of $(B-V)$ together with a curve based primarily on angular diameters, with extrapolation to lower temperatures by various procedures. It is assumed for the nearby binaries that interstellar absorption and reddening can be neglected.

Popper (1998) has found that some EB components lie close to the flux curve and others depart from the curve by substantial amounts (see his Fig. 1). For 4 systems Popper explains this departure by intrinsic variability of these stars as their light curves show considerable irregularities, presumable caused by large spotted areas. But for one system (HS Hya), also departing from the flux curve substantially, there is no evidence for intrinsic variation in the light of the components.

Using our assumption that observational parameters of EB stars, belonging to Popper's sample, differ from those of isolated stars (used for the construction of his flux curve) we can draw the following conclusions from Popper's Fig. 1:

- Main sequence EB components in the spectral range F5-K3 $(B-V>0.4)$ have larger radii in comparison with isolated stars; this, according to Eq. (2), leads to smaller fluxes.

- However, the 4 hottest stars in the Popper's sample (components of $\zeta$ Phe and $\beta$ Aur, spectral range B6-A1) show larger fluxes that, according to Eq. (2), can be a result of their smaller radii.

\subsection{Bolometric corrections}

Visual binaries with known distances allow us to estimate $v i$ sual luminosities of the components, while for double-lined 
eclipsing binaries bolometric luminositites of the components can be calculated from their $T_{\text {eff }}$ and radii.

So, to match the two mass-luminosity relations we need bolometric corrections $(B C)$, the difference between bolometric absolute magnitude and visual absolute magnitude. The bolometric corrections depend on the physical conditions in the stellar outer layers.

There are dozens of $B C$ scales published in the literature. As their comparison shows (see, e.g., Flower's 1996 Fig. 9b; Malkov et al.'s 1997 Table 3 and Fig. 1) they are all in good agreement, but the Habets \& Heintze (1981) scale deviates significantly from other scales, showing smaller bolometric corrections for almost all spectral range, from mid-B to late-M types.

There were numerous attempts to explain the Habets \& Heintze (1981) scale deviations in the literature: by overestimation of low-mass stellar radii (Haywood 1993), by erroneous determination of the Sun's bolometric correction (Flower 1996), by a lack of cool stars and a very rough approximation of the MLR and other relations used in their study (Malkov et al. 1997). But the difference between components of eclipsing binaries and components of visual binaries can be another reason for the deviation of the Habets \& Heintze (1981) $B C$ scale.

Most of the published $B C$ scales are based on photometric data. However, to construct their $B C$ scale, Habets \& Heintze (1981) used bolometric magnitudes that had been calculated from radii and effective temperatures of binary stars.

Habets \& Heintze (1981) collected all published data for both visual and eclipsing binaries. Observational data for visual binaries with known parallaxes yields mass $(m)$ and visual absolute magnitude $M_{V}$ of the components. The $\left[m-M_{V}\right]$ relation was then constructed by the authors from this data.

On the other hand, masses, radii $(R)$ and spectra $(S p)$ of the components of eclipsing binaries were used by the authors to construct $[m-R]$ and $[m-S p]$ relations. A spectrumtemperature calibrating relation based on various publications was adopted by Habets \& Heintze and used to transform their $[m-S p]$ relation to a $\left[m-T_{\text {eff }}\right]$ one. The latter, coupled with the $[m-R]$ relation, was used to give a $\left[m-M_{\mathrm{bol}}\right]$ relation, where $M_{\text {bol }}$ is the bolometric absolute magnitude calculated from $T_{\text {eff }}$ and $R$ according to the Stefan-Boltzmann law.

The $\left[m-M_{V}\right]$ relation based on the visual binaries data and the $\left[m-M_{\mathrm{bol}}\right]$ relation based on the eclipsing binaries data produced the $[m-B C]$ relation.

Since the Habets \& Heintze's (1981) procedure of the construction of their $B C$ scale is based on coupling data for EB components and isolated stars (visual binary components), the comparison of the Habets \& Heintze (1981) BC scale with other (photometric) $B C$ scales can itself serve as a test for differences between these two types of stars.

As was mentioned above, the Habets \& Heintze (1981) scale deviates significantly (always showing smaller bolometric corrections) from other scales for almost the whole spectral range.

The visual binary set of Habets \& Heintze almost does not contain well-resolved main sequence components of masses larger than 1.5 (hereafter all masses are given in solar units). Few exceptions are Sirius A $(m=2.14), \zeta$ Sge A and B
Table 1. Radii and effective temperatures of components of main sequence eclipsing binaries in comparison with those of isolated stars.

\begin{tabular}{llll}
\hline \hline$R$-test & $T$-test & $F$-test & $B$-test \\
\hline \multicolumn{5}{c}{ A-F-G-K stars } \\
A2-F9 & A0-F8 & F5-K3 & A1-G2 \\
$R \nearrow$ & $R \nearrow$ & $R \nearrow$ & $R \nearrow$ \\
& or/and $T_{\text {eff }} \nearrow$ & or/and $T_{\text {eff }} \nearrow$ \\
\hline \multicolumn{4}{c}{ B-stars } \\
B0-A1 & B6-A1 \\
$R \searrow$ & \multicolumn{4}{c}{$R \searrow$} \\
or/and $T_{\text {eff }} \nearrow$ & \multicolumn{4}{c}{} \\
\hline
\end{tabular}

$R$-test: radius comparison (Fig 1); $T$-test: $T_{\text {eff }}$ comparison (Ribas et al., 1998); $F$-test: flux comparison (Popper, 1998); $B$-test: comparison of Habets \& Heintze (1981) $B C$ scale with other scales.

( $m=4.0$ and 2.0) and ADS $11871 \mathrm{~A}(m=2.28)$. But, according to Heintz (1981), $\zeta$ Sge A is a spectroscopic binary; and ADS 11871 shows likely variable radial velocity (Tokovinin \& Smekhov 2002). On the other hand, only few reliable eclipsing binaries with $m<1$ are known. All of this means that the Habets \& Heintze $B C$ scale for stars earlier than $\sim \mathrm{A} 1$ and later than $\sim \mathrm{G} 2$ is a result of extrapolation.

The following conclusion can be drawn from the comparison of the $B C$ scales: A1 to G2 $\mathrm{EB}$ components have (in comparison with isolated stars) larger radii or/and higher temperatures. Both reasons lead to an increase of the bolometric luminosity and, according to the Habets \& Heintze's (1981) procedure, to an increase of the bolometric correction.

Results of the current section are summarized in Table 1.

\section{Discussion}

The results of a comparison of observational parameters of eclipsing binaries and isolated stars made in the previous section show that

- at least for early-A to late-F spectral types EB components have larger radii (or/and higher effective temperatures) than isolated stars;

- EB components of B-stars have, on average, $20 \%$ smaller radii than isolated stars.

Here only results are listed which are confirmed by at least two independent tests. Below we give some possible explanations for the observed difference in radius and temperature.

\subsection{Synchronization and slowing down of EB components}

As is well-known, isolated early-type stars are rapid rotators. But tidal forces produce synchronous rotation in close binaries, and almost all pairs with period less than $15^{\mathrm{d}}$ are synchronized, so components of close binaries rotate more slowly. Figure 2 illustrates this statement. The line approximates the rotation 


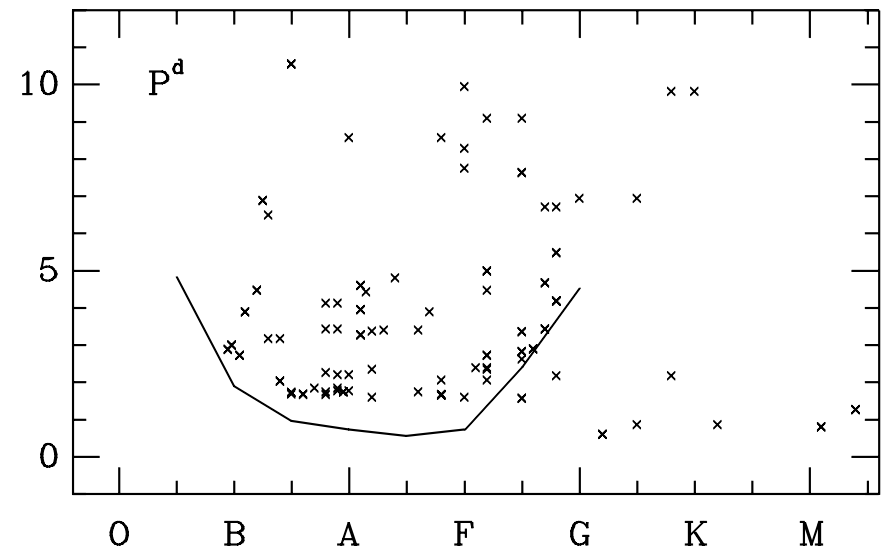

Fig. 2. Rotation period of isolated stars (line, McNally 1965) and orbital period of eclipsing binaries (points, Malkov 1993) as a function of spectral type.

period - spectrum relation for single stars from McNally (1965), points represent orbital periods of EB stars from the Catalogue of astrophysical parameters of binary systems by Malkov (1993). One can see that for spectral classes F and earlier rotational periods of close pair components are systematically larger than those of isolated stars (if we suppose all these close pairs to be synchronized).

Stellar rotation changes stellar evolution and the global parameters of a star (see, e.g., the comprehensive review of Maeder \& Meynet 2000). Centrifugial forces partially compensate the force of gravity; temperature and pressure in the central parts of a star are smaller, consequently the energy production rate is also decreasing. It means that rapid rotators have lower total luminosity and lower temperature; and they evolve more slowly. On the other hand, if EB components rotate more slowly, they should have higher temperatures and luminosities. Such a star should evolve with a higher rate than a rapid rotator, i.e., its radius on the main sequence should increase more rapidly than that of a rapidly rotating isolated star.

\subsection{Selection effect: orientation of EB components}

Due to the non-spherical shape of rotating stars, the observed effective temperature, radius and magnitude depend on the line of sight. Isolated stars are randomly oriented; EB components are mainly observed from the equator (see, e.g., Abt 2001 for discussion). This can explain why their observational radii and temperatures systematically differ from those of isolated stars. Observers tend to approximate the observational radius with an unperturbed radius (i.e., the radius of a sphere with the same volume as the component), but these rectifying methods introduce systematic errors in the derived elements (see, e.g., Söderhjelm 1974). This effect also may cause differences between observational parameters of close pair components and those of isolated stars of spectral types $F$ and earlier.

\subsection{Radii of B-type EB components}

The smaller observational radii of B-type EB components can be explained as follows.

Relative radii ( $r=R / a$, where $a$ is the semi-major axis) in B-type systems are, on average, larger than in pairs of later spectral types; a relatively small increase of a component's radius $R$ (due to evolution) leads to its Roche lobe filling (that arrests the star's further growth in size), moves the system to "semi-detached" and thus excludes it from the statistics of detached main sequence binaries. On the other hand, systems with larger $a$ (sufficient to keep a "detached" status) have lower probability of discovery.

\section{Mass-luminosity relation for intermediate and high masses}

It was repeatedly stated that the present-day knowledge of the MLR and the luminosity function at the faintest magnitudes is not sufficient for drawing definite conclusions on the IMF behaviour at low masses. But our results lead to the conclusion that the IMF for larger masses should also be revised.

Indeed, visual binaries for which masses are known to an acceptable accuracy are, with few exceptions, nearby stars (parallaxes less than 0.1 are of little use in mass determination), and consequently have spectra later than G0 (see discussion on massive visual binaries in Sect. 2.4). On the other hand, the majority of detached main-sequence double-lined eclipsing binaries are systems earlier than G2 (later systems, as a rule, are not bright enough to provide reliable spectroscopic data). It means, particularly, that present-day empirical knowledge of the MLR for $m \geq 1.5$ is based exclusively on high accuracy data for the main sequence components of double-lined EB stars (see Fig. 3, top panel).

However, if we assume these stars to have different observational parameters (radius, temperature and consequently luminosity) from isolated stars, the resulting MLR cannot be applied to derive a single stars IMF.

For this purpose, the MLR can be obtained only from the data on visual binaries. But the number of points in Fig. 3 (middle panel), representing ground-based observations for visual binaries with masses larger than 1.5 , as well as the accuracy of these data are clearly insufficient to work out the shape of the MLR in this mass range. And even the data from Hipparcos have not improved the situation significantly (Fig. 3, bottom panel).

Thus, we find that the empirical mass-luminosity relation for stars with masses larger than 1.5 (based exclusively on data for EB components) evidently cannot be applied to derive the IMF. On the other hand, empirical data for components of wide visual binaries with $m \geq 1.5$ is very poor.

It should be recalled, in addition, that not only the general behaviour of the MLR but its precise shape (slope) is crucial for deriving the initial mass function from observational luminosity function, as the relation

$\frac{\mathrm{d} N}{\mathrm{~d} \log m}=\frac{\mathrm{d} N}{\mathrm{~d} M_{V}} *\left|\frac{\mathrm{d} M_{V}}{\mathrm{~d} \log m}\right|$ 


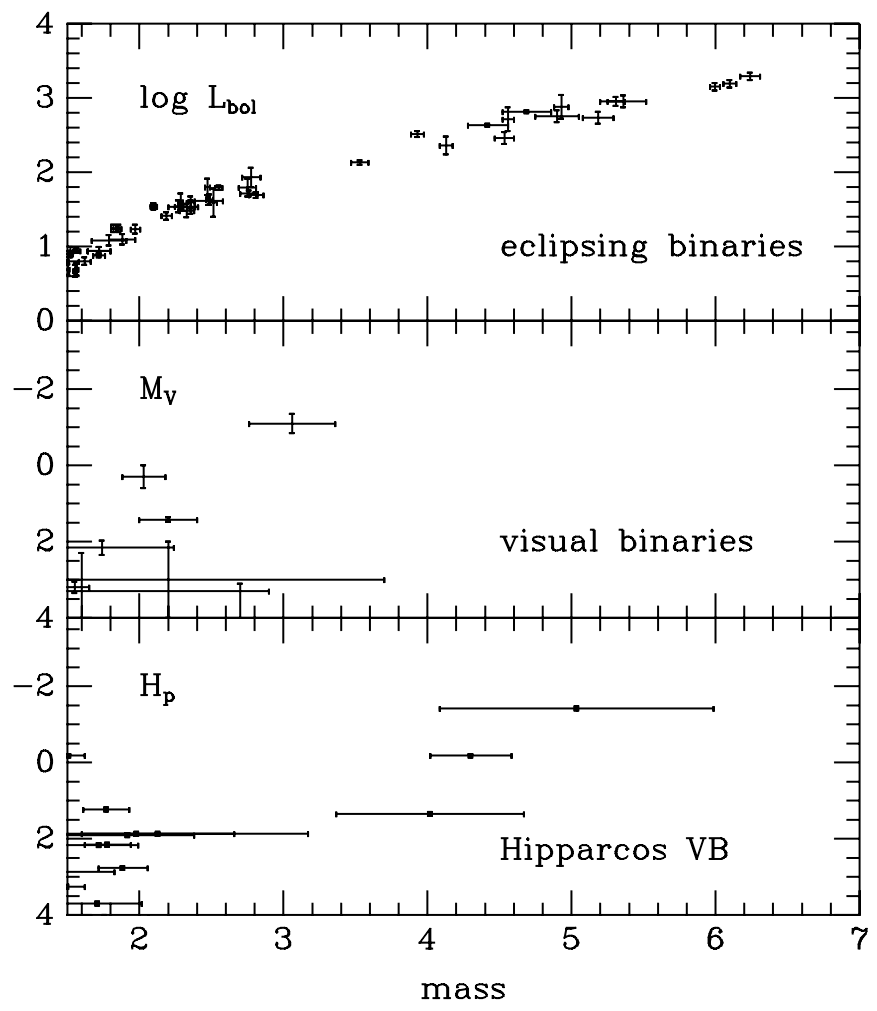

Fig. 3. Mass-luminosity relation for main sequence stars. From top to bottom: eclipsing binaries with accurate (better than 3-5\%) masses; ground based data for visual and resolved spectroscopic binaries; Hipparcos data for visual binaries (Martin \& Mignard 1998; Söderhjelm 1999).

contains the MLR's derivative that requires the knowledge of the MLR fine structure.

Qualitatively, one can expect the following changes in the IMF shape if our assumptions are correct.

It should be mentioned first that for stars more massive than $m \sim 10$ or so the IMF is not derived from the MLR. Stellar isochrones and spectra are used instead (see, e.g., Massey et al. 2000).

Isolated B-type stars, on average slightly more evolved than B-type EB components, should basically show the same (or slightly larger) luminosities for a given mass, as their evolutionary tracks at this stage are almost horizontal in the HRD. It means that one cannot expect significant changes in the MLR and, consequently, no changes in the IMF for stars with masses more than $\sim 3$ are expected.

However, the luminosities of A- and F-type isolated stars, according to our estimates, should be less than the luminosities of EB components of the corresponding masses. It means that a corrected MLR should lie lower than the EB-MLR in the mass-luminosity plane, beginning from early A towards cooler stars. This, coupled with the assumption on insignificant (if any) changes in B-type region (see above), leads to a steeper shape of the corrected MLR at least for A-type stars. The IMF becomes larger due to a larger second multiplier on the right side of Eq. (3).

Also, smaller luminosities for a given mass cause larger values of the luminosity function (which is ascending for these magnitudes), the first multiplier on the right side of Eq. (3). This is another reason for larger values of the IMF (at least for late-A and $\mathrm{F}$ stars).

According to various publications (Kroupa et al. 1993; Scalo 1998) the IMF changes its slope somewhere around one solar mass and becomes steeper for larger masses. If our estimations are to be correct, this "break-point" should be shifted to $m \sim 1.5$. On the other hand, at $m \sim 2-2.5$ the IMF should be steeper than presently derived.

New observations of high accuracy are necessary to make more definite conclusions on this important subject. A focus for visual binaries astrometry with future space missions (DIVA, GAIA) must be to observe a few hundred wide systems with $m>1.5$. Their masses should be determined with uncertainties better than $3 \%$. Also accurate photometry should be provided to obtain visual luminosities.

\section{Conclusions}

We can draw the following conclusions.

Comparing the radii of eclipsing binaries components and single stars we have found a noticeable difference between observational parameters of B0V-G0V components of eclipsing binaries and those of single stars of the corresponding spectral type. This difference was confirmed by re-analysing the results of independent investigations published in the literature. This difference explains a disagreement between published scales of bolometric corrections.

Larger radii and higher temperatures of A-F eclipsing binaries can be explained by synchronization of such stars in close systems that prevents them to rotate rapidly. Observational selection effect can also cause such features: due to the nonspherical shape of rotating stars, the observed parameters depend on the line of sight; isolated stars are randomly oriented, but eclipsing binary components are mainly observed from the equator.

The smaller observational radii of B-type eclipsing binaries can be explained by the absence of stars with larger radii among detached main sequence pairs: the majority of them filled their Roche lobe (that arrested their further growth in size) and became semi-detached systems (that excludes them from the statistics).

So, we have found that the mass-luminosity relation based on eclipsing binary data cannot be used to derive the initial mass function of single stars.

While our current knowledge of the empirical massluminosity relation for intermediate-mass ( 1.5 to $\left.10 m_{\odot}\right)$ stars is based exclusively on data from eclipsing binaries, knowledge of the mass-luminosity relation should come from dynamical mass determinations of visual binaries, combined with spatially resolved precise photometry.

Then the initial mass function should be revised for $m>$ $1.5 m_{\odot}$.

Acknowledgements. I acknowledge Dana Kovaleva and Hans Zinnecker for very fruitful discussions. I thank John Scalo for his useful suggestions for clarifying the paper. I acknowledge support from the RF President grant 09-15-96022. 


\section{References}

Abt, H. A. 2001, AJ, 122, 2008

Delfosse, X., Forveille, T., Ségransan, D., et al. 2000, A\&A, 364, 217

Flower, P. J. 1996, ApJ, 469, 355

Gorda, S. Yu., \& Svechnikov, M. A. 1998, Astron. Rep., 42, 793

Habets, C. M. H. J., \& Heintze, J. R. W. 1981, A\&AS, 46, 193

Haywood, M. 1993, A\&A, 282, 444

Heintz, W. D. 1981, ApJS, 46, 247

Henry, T. J., Franz, O. G., Wasserman, L. H., et al. 1999, ApJ, 512, 864

Kovaleva, D. A. 2001, Astron. Z., 78, 1104

Kroupa, P., Tout, C. A., \& Gilmore, G. 1993, MNRAS, 262, 545

Maeder, A., \& Meynet, G. 2000, ARA\&A, 38, 143

Malkov, O. Yu. 1993, Bull. Inf. CDS, 42, 27

Malkov, O. Yu., Piskunov, A. E., \& Shpil'kina, D. A. 1997, A\&A, 320,79
Martin, C., \& Mignard, F. 1998, A\&A, 330, 585

Massey, P., Waterhouse, E., \& DeGioia-Eastwood, K. 2000, AJ, 119, 2214

McNally, D. 1965, The Observatory, 85, 166

Pasinetti-Fracassini, L. E., Pastori, L., Covino, S., \& Pozzi, A. 2001, A\&A, 367, 521

Popper, D. M. 1998, PASP, 110, 919

Ribas, I., Gimenez, A., Torra, J., Jordi, C., \& Oblak, E. 1998, A\&A, 330,600

Scalo, J. 1998, in The Stellar Initial Mass Function, ed. G. Gilmore, \& D. Howell, ASP Conf. Ser., 142, 201

Söderhjelm, S. 1974, A\&A, 34, 59

Söderhjelm, S. 1999, A\&A, 341, 121

Tokovinin, A. A., \& Smekhov, M. G. 2002, A\&A, 382, 118 